\title{
EMDR y el Modelo del Procesamiento Adaptativo DE LA Información
}

\section{Mecanismos potenciales del cambio}

\author{
Roger M. Solomon \\ Critical Incident Recovery Resources, Williamsville, NY \\ Francine Shapiro \\ Mental Research Institute, Menlo Park, CA
}

\begin{abstract}
La desensibilización y el reprocesamiento a través de movimientos oculares (EMDR) es un enfoque terapéutico guiado por el modelo del procesamiento adaptativo de la información (PAI). Este artículo ofrece una breve visión general de algunos de los principales preceptos del PAI. Se formula la hipótesis de que la base de la patología clínica son los recuerdos almacenados disfuncionalmente, produciéndose el cambio terapéutico a través del procesamiento de dichos recuerdos dentro de redes adaptativas más amplias. A diferencia de las terapias de exposición basadas en la extinción, se propone que los recuerdos sobre los que se incide en EMDR se transmutan durante el procesamiento, volviéndose a almacenar por medio de un proceso de re-consolidación. Por tanto, se ofrece comparación y contraste con los modelos de procesamiento de la información y tratamientos basados en la extinción, incluyendo las implicaciones para la práctica clínica. A lo largo del artículo, se habla de diversos mecanismos de acción, incluyendo aquellos derivados de los principios del modelo PAI, así como los propios procedimientos de EMDR, incluyendo la estimulación bilateral. Se ofrecen sugerencias de investigación, con el fin de investigar diversas hipótesis.
\end{abstract}

Palabras Clave: EMDR; modelo de procesamiento adaptativo de la información; mecanismos de cambio; procesamiento de información; psicoterapia

$\mathbf{L}$ a desensibilización y reprocesamiento por movimientos oculares (EMDR) es un enfoque terapéutico que enfatiza el sistema de procesamiento de la información intrínseco del cerebro y cómo se almacenan los recuerdos. Los síntomas actuales son vistos como el resultado de experiencias perturbadoras que no han sido procesadas adecuadamente y han sido codificadas de manera disfuncional, específica del estado (Shapiro, 1995, 2001, 2007a). La esencia de EMDR implica la transmutación de estas experiencias disfuncionalmente almacenadas hacia una resolución adaptativa que promueve la salud psicológica. Para que se pueda aplicar EMDR de modo eficaz, el clínico necesita un marco de trabajo que identifique recuerdos diana apropiados y un orden de procesamiento para obtener efectos óptimos del tratamiento. El modelo de procesamiento adaptativo de la información (PAI), que informa el tratamiento EMDR, contiene una variedad de principios y predicciones que implican diversos agentes de cambio potenciales. El examen exhaustivo de todos los principios del PAI está más allá del alcance de este artículo (ver Shapiro 2001, 2006). Sin embargo, debido a que el EMDR es un enfoque complejo con muchos

This article originally appeared as Solomon, R. M., \& Shapiro, F. (2008). EMDR and the Adaptive Information Processing Model: Potential Mechanisms of Change. Journal of EMDR Practice and Research, 2(4): 315-325. Translated by Miriam Ramos Morrison 
elementos, el propósito de este artículo es resaltar un rango de posible agentes de cambio, además del movimiento ocular y otros tipos de estimulación bilateral que han recibido mayor atención.

El artículo comienza con una breve visión general del modelo PAI y la base propuesta de la patología clínica. Se discute la transmutación observada de los recuerdos procesados, junto con conjeturas relacionadas con las recientes investigaciones sobre la reconsolidación de la memoria, que es un proceso neurobiológico sobre el que se plantea la hipótesis de que subyace a los efectos de EMDR. Ya que se cree que la reconsolidación difiere de la extinción en términos de los procesos neurobiológicos implicados, se exploran las similitudes y diferencias entre el modelo PAI y las terapias de exposición basadas en la extinción, junto con implicaciones para la práctica clínica. Se proponen investigaciones para comprobar tanto los principios como los potenciales mecanismos de acciones. Después se consideran los potenciales mecanismos de acción encargados de los procedimientos de EMDR, incluyendo la estimulación bilateral. Ha de señalarse que, aunque abundan las teorías, se desconocen con precisión los mecanismos de cambio en cualquier tipo de terapia, siendo necesarios estudios aleatorios para una exploración y delineación completa. Por tanto, se ofrecen sugerencias de futuras investigaciones para diversas hipótesis.

\section{Modelo PAI}

El modelo PAI explica las bases de la patología, predice resultados clínicos de éxito y guía la conceptualización del caso y los procedimientos de tratamiento. El modelo PAI, en concordancia con otras teorías del aprendizaje, postula la existencia de un sistema de procesamiento de la información que asimila nuevas experiencias en redes de memoria ya existentes. Estas redes de memoria son la base de la percepción, las actitudes y el comportamiento. Las percepciones de situaciones actuales están automáticamente enlazadas con redes de memoria asociadas (Buchanon, 2007). Por ejemplo, el lector puede darle sentido a esta frase debido a experiencias previas con el lenguaje escrito ${ }^{1}$. De modo similar, el quemarse una mano en un hornillo se dirige a las redes de memoria relacionadas con hornillos y el peligro potencial de los objetos calientes. Un conflicto con un compañero de juegos ("yo primero") y su resolución ("podemos compartir") se acomoda y

\footnotetext{
${ }^{1}$ Nota del traductor: En el original, “inglés escrito”. Se generaliza a "lenguage escrito" para mayor universalidad.
}

asimila en redes de memoria que tienen que ver con las relaciones y se añade a la base de conocimientos disponible relacionada con relaciones interpersonales y resolución de conflictos. Cuando trabaja adecuadamente, el sistema innato de procesamiento de información "metaboliza" o "digiere" nuevas experiencias. Las percepciones sensoriales entrantes se integran y conectan con información relacionada que ya se encuentra almacenada en redes de memoria, permitiéndonos darle sentido a nuestra experiencia. Aquello que es útil se aprende, se almacena en redes de memoria con las emociones apropiadas y queda disponible para guiar a la persona en el futuro (Shapiro, 2001).

\section{Patología según el modelo PAI}

Los problemas surgen cuando una experiencia se procesa de forma inadecuada. El modelo PAI de Shapiro $(1995,2001,2006)$ postula que un incidente particularmente angustiante puede quedar almacenado de manera específica a cada estado, lo que quiere decir que se mantiene congelado en el tiempo en su propia red neural, incapaz de conectar con otras redes de memoria que contienen información adaptativa. La Dra. Shapiro formula la hipótesis de que cuando un recuerdo está codificado de forma específica a un estado, excitador y angustiante, las percepciones originales pueden continuar siendo desencadenadas por una serie de estímulos internos y externos, lo que resulta en reacciones emocionales, cognitivas y conductuales inapropiadas, así como síntomas manifiestos (por ejemplo, ansiedad alta, pesadillas, pensamientos intrusivos). Se entiende que los recuerdos almacenados disfuncionalmente sientan las bases de futuras respuestas no adaptativas, porque las percepciones de las situaciones actuales están automáticamente enlazadas con redes de memoria asociadas. Los eventos de la infancia también pueden estar codificados con mecanismos de supervivencia e incluir sensaciones de peligro que son inapropiadas para los adultos. Sin embargo, estos eventos pasados mantienen su fuerza porque, con el tiempo, no han sido asimilados de manera adecuada dentro de redes adaptativas.

El modelo PAI considera las conductas negativas y las características de personalidad como el resultado de información que se mantiene de manera disfuncional (Shapiro, 2001). Desde esta perspectiva, una auto-creencia negativa (por ejemplo, "No soy suficientemente bueno") no es considerada la causa de la disfunción presente; se entiende como un síntoma de las experiencias previas no procesadas que contienen esa emoción y perspectiva. Las actitudes, emociones y sensaciones no son consideradas como simples 
reacciones ante un evento pasado; son vistas como manifestaciones de las percepciones almacenadas fisiológicamente en la memoria y las reacciones ante ellas. Esta visión de los síntomas presentes como el resultado de la activación de recuerdos que han sido procesados y almacenados de modo inadecuado es esencial en el tratamiento EMDR. Como tal, la reestructuración de creencias y la manipulación conductual dirigidas no se consideran dentro del modelo PAI como los agentes de cambio, dado que están contempladas en otros tratamientos. La realización de investigaciones que evalúen los mecanismos que dan lugar a los cambios progresivos en creencias y autoconfianza que conlleva el procesamiento EMDR, en comparación con otros tratamientos, puede ayudar a clarificar este asunto.

\section{Transmutación de la memoria}

Al ver la patología como el resultado de experiencias no procesadas, el modelo PAI (Shapiro, 1995, 2001, 2006) considera que las experiencias procesadas son la base de la salud mental. El protocolo EMDR implica acceder a la información almacenada disfuncionalmente, estimular el sistema de procesamiento innato a través de procesos y procedimientos estandarizados (incluyendo la estimulación bilateral), y facilitar enlaces dinámicos a las redes de memoria adaptativas, permitiendo por tanto que cambien las características del recuerdo a medida que se transmuta hacia una resolución adaptativa. La transcripción de sesiones (Shapiro, 2001, 2002; Shapiro y Forrest, 1997) indica que el procesamiento ocurre generalmente a través de una progresión rápida de conexiones intrapsíquicas en la sesión a medida que las emociones, "insights", sensaciones y recuerdos salen a la superficie y cambian con cada nueva tanda de estimulación bilateral. Los mecanismos de acción propuestos incluyen la asimilación de información adaptativa encontrada en otras redes de memoria que enlazan con la red en la que se encuentra el evento perturbador que estaba previamente aislado. Después de un tratamiento con éxito, se postula que el recuerdo ya no se encuentra aislado, porque parece estar integrado de manera apropiada dentro de la red de memoria más amplia. Por tanto, se entiende que el procesamiento implica la forja de nuevas asociaciones y conexiones que permiten que tenga lugar el aprendizaje con el recuerdo almacenado de manera nueva y adaptativa.

Como señala Shapiro $\left(2007^{\mathrm{a}}\right)$, la hipótesis PAI parece estar en concordancia con las teorías neurobiológicas recientes de la reconsolidación de la memoria (Cahill y McGaugh, 1998; Suzuki et al., 2004), que proponen que un recuerdo al que se ha accedido puede volverse voluble y restablecerse de un modo modificado. Como indican Suzuki y colegas (2004), parece que la reconsolidación y la extinción tienen mecanismos neurobiológicos claramente diferentes. Mientras que se piensa que la reconsolidación altera el recuerdo original, los procesos de extinción parecen crear un nuevo recuerdo que compite con el antiguo. Esto tiene implicaciones particulares para los modelos y terapias de exposición basados en la extinción (por ejemplo, Brewin, 2006; cf. McCleery y Harvey, 2004). La base neurobiológica de la extinción ha sido relacionada con la actividad en un receptor concreto de la amígdala, y se ha investigado usando cierto compuesto que se sabe activa ese receptor y aumenta la extinción con el fin de examinar el mecanismo de extinción en terapias basadas en la exposición para la acrofobia y la ansiedad social (Hofmann et al., 2006; Ressler et al., 2004). Desafortunadamente, parece que los compuestos también aumentan la reconsolidación (Lee, Milton y Everitt, 2006). Sin embargo, la investigación también ha indicado que "el antagonismo farmacológico del receptor canabinoide 1 o de los canales de calcio regulados por el voltaje de tipo L bloquean la extinción pero no la reconsolidación" (Suzuki et al., 2004, p. 4787). Este tipo de investigación que utiliza estos compuestos (por ejemplo, Rimonabant) determinaría de manera más definitiva si la reconsolidación es el mecanismo primario subyacente a los efectos de EMDR.

Otra investigaciones sugeridas consisten en estudios controlados comparando terapias basadas en la extinción y EMDR para investigar (1) los tipos de asociaciones disponibles para el cliente antes y después del tratamiento; (2) las diferencias en la capacidad de acceder a recuerdos visuales precisos del recuerdo original; y (3) las diferencias en tasas de recaída, que puede que aclaren estas posibilidades. En concreto, no se esperaría que los efectos de la extinción se generalizasen a un nuevo evento posterior al tratamiento que fuese muy similar al incidente crítico original. Sin embargo, informes recientes de casos indican que el tratamiento EMDR se generaliza a eventos futuros (por ejemplo, Shapiro, Kaslow y Maxfield, 2007), lo que sugiere un mecanismo de reconsolidación, más que de extinción. EMDR puede ayudar a fomentar resiliencia y a que no haya recaídas cuando los clientes se enfrentan a un trauma similar (Rost, Hoffman y Wheeler, en prensa; Zaghout-Hodali, Alissa y Dodgson, 2008). Hace falta investigación para hacer un seguimiento sistemático de individuos tratados tanto con tratamientos de EMDR como con exposición prolongada para determinar si hay diferencia en 
las respuestas de los participantes a los traumas posteriores al tratamiento. Esto sería un modo simple de examinar y comparar las predicciones y resultados de los modelos de extinción y reconsolidación.

\section{Similitudes y diferencias de otros modelos de procesamiento de la información}

El modelo PAI, en ciertos aspectos, concuerda con el modelo de procesamiento emocional que subyace a los tratamientos basados en la exposición más utilizados. De forma abreviada, Foa y Kozak (1986) sugieren que, para que tenga lugar la reducción del miedo, han de cumplirse dos condiciones. En primer lugar, debe haber activación del recuerdo del miedo. Segundo, debe proporcionarse información correctiva con elementos incompatibles con la estructura del miedo para que pueda formarse un nuevo recuerdo. La incorporación de la nueva información da como resultado una reducción de las respuestas de miedo (a través de la habituación en las sesiones y entre sesiones), permitiendo cambios en el significado de la experiencia.

El modelo PAI es concordante en la medida en que los procedimientos y protocolos facilitan el acceso de redes emocionales y la incorporación de nueva información (Rogers y Silver, 2002). Se considera que la información correctiva en las terapias basadas en la exposición, tal como la exposición prolongada (por ejemplo, Foa, Rothbaum, Riggs y Murdock, 1991; Rothbaum, Astin y Marsteller, 2005), proviene de la situación terapéutica y el efecto de habituación (Foa y Kozak, 1986; Rothbaum et al., 2005). Sin embargo, los cambios que tienen lugar en EMDR sugieren que los clientes incorporan información no sólo del contexto terapéutico, sino también de recuerdos de experiencias anteriores en la vida (Shapiro, 1995, 2001, 2007b). El enlace de información dentro de y entre recuerdos parece ser espontáneo, sin ser provocado por el terapeuta, y no el resultado de una exposición al recuerdo repetida y mantenida. Rogers y Silver (2002) concluyeron que EMDR parece concordar con el proceso de asimilación y acomodación y de procesamiento de la información, más que con el de habituación. Estas observaciones, aunque especulativas, concuerdan con el hecho de que el recuerdo diana se almacene de forma adaptativa debido a la reconsolidación, más que con que los cambios tengan lugar por la formación de un nuevo recuerdo. Una vez más, la investigación que compara el rememorar recuerdos originales y las tasas y tipos de patrones de recuperación puede aclarar si los mecanismos primarios de acción en EMDR están basados en la extinción o si son principalmente mecanismos que consisten en asociación, asimilación y reconsolidación. Además, los análisis de los procesos, tal y como los llevados a cabo por Lee, Taylor y Drummond (2006), Rogers et al. (1999), y McCullough (2002) pueden ayudar a clarificar los mecanismos concretos.

\section{Modelos y práctica clínica}

Aunque otros modelos de procesamiento de la información también están basados en conceptos de redes de memoria, cada modelo enfatiza aspectos y consideraciones diferentes. Los diversos modelos guían las prácticas de sus tratamientos propuestos y consideran que los agentes de cambio son elementos diferentes. Por ejemplo, como se ha señalado anteriormente, el modelo PAI coincide con la noción de que el procesamiento implica la incorporación de "información correctiva" (Foa y Kozak, 1986). Sin embargo, no considera el cambio en valoración cognitiva como el determinante clave. En lugar de ello, el modelo PAI considera el procesamiento como una integración del recuerdo almacenado disfuncionalmente dentro de redes ya existentes que contienen información adaptativa. De ahí que haga hincapié en la necesidad de que existan redes de recuerdos positivos para que el procesamiento tenga lugar. Por tanto, la recogida de historia consiste en evaluar si existen las redes positivas e incorporarlas si no existen. Este principio también guía la práctica clínica de EMDR si el procesamiento se atasca durante una sesión de tratamiento. En ese caso, el clínico imita el procesamiento espontáneo accediendo deliberadamente a la siguiente red positiva que esté disponible en la historia del cliente o inculca la información necesaria para formar una red positiva que pueda enlazarse.

Los dos modelos también difieren en la visión de la sintomatología actual. El modelo PAI no considera que la fuente principal de la disfunción del cliente sean las respuestas condicionadas, la reacción emocional actual a un evento pasado, ni la valoración cognitiva de un evento pasado. Más bien, el modelo PAI considera que el problema está causado por las percepciones almacenadas fisiológicamente (imágenes, pensamientos, creencias, emociones, sensaciones, olores, etc.) del evento pasado. Por tanto, al contrario que otros modelos, una predicción basada en principios PAI diría que procesar recuerdos principales elimina las percepciones disfuncionales del almacenamiento. Por ejemplo, el modelo PAI predice que muchas de las sensaciones que componen el dolor de un miembro fantasma están actualmente almacenadas en la memoria y pueden ser eliminadas por 
medio del procesamiento de recuerdos salientes (por ejemplo, Russell, 2007; Schneider, Hoffman, Rost y Shapiro, 2007, 2008; Shapiro, 2001; Wilensky, 2006). No se cree que el principal agente de cambio sea la exposición prolongada, la atención enfocada y extensa en el evento, ni los cambios en valoración cognitiva. En lugar de ello, el cambio es considerado como un subproducto del procesamiento, que está causado por el proceso de asociación interna.

Esto no quiere decir que el condicionamiento no exista, ni que las valoraciones cognitivas no seas significativas. Por ejemplo, la perturbación actual es tratada en la terapia EMDR a través de procesar primero el trauma más temprano. De hecho, los informes clínicos indican que, subsecuentemente al procesamiento del evento pasado, el disparador identificado inicialmente frecuentemente deja de ser perturbador (Shapiro et al., 2007). Sin embargo, la segunda vertiente del tratamiento EMDR implica procesar directamente el disparador, porque nuevos estímulos pueden volverse perturbadores de forma autónoma a través del condicionamiento de segundo orden. Sin embargo, se asume que estos eventos condicionantes han sido almacenados en la memoria y pueden ser adecuadamente tratados a través del procesamiento. No debería asumirse que cada enfoque terapéutico tendrá mecanismos de cambio completamente diferentes, ni que haya solo uno. La complejidad de cualquier tratamiento aumenta el número potencial de mecanismos de cambio que interactúan para causar efectos de tratamiento positivos. Sin embargo, la investigación que examina en detalle las predicciones de los diversos modelos puede no solo verificar los principios, sino que puede ser capaz de ofrecer información sobre los posibles mecanismos de cambio.

\section{Evaluaciones de tratamiento}

Determinar los mecanismos de acción de cualquier terapia es un proceso complejo, porque implica múltiples niveles de observación y análisis. Las hipótesis pueden variar desde constructos como estados mentales (ver sección "Mindfulness"), características específicas de procesamiento de la información en general (por ejemplo, conjeturas respecto a la configuración de las redes de memoria y sus interacciones); los tipos específicos de procedimientos utilizados para evocar el cambio (por ejemplo, exposición prolongada, estimulación bilateral); los procesos subyacentes que han sido postulados (por ejemplo, extinción, inhibición transmarginal, respuesta de orientación, interrupción de la memoria funcional); los concomitantes fisiológicos específicos (por ejemplo, reducciones en neurotransmisores específicos); o la interacción de diversas estructuras cerebrales. Las evaluaciones de la investigación de una variedad de principios del PAI ofrecen una fructífera rampa de lanzamiento para estas investigaciones. Aunque la investigación preliminar ha ofrecido apoyo a diversas hipótesis, se necesita una investigación controlada.

Como se ha señalado anteriormente, una premisa principal del modelo PAI es que la fuente de la patología y la salud son las redes de recuerdos almacenados fisiológicamente. La patología es vista como recuerdos no procesados y el modelo PAI predice que el procesamiento resultará en una disminución o eliminación de la sintomatología. Aunque esto ha sido ampliamente aceptado en el tratamiento de TEPT por medio del tratamiento del evento crítico (criterio A), el modelo PAI postula que la mayoría de las formas de patología también están basadas en recuerdos no procesados y pueden resolverse permitiendo que los recuerdos que causan el problema lleguen a una resolución adaptativa. Esta predicción está apoyada por un amplio rango de estudios de casos que indican que problemas tan diversos como el trastorno dismórfico corporal (Brown, McGoldrick y Buchanan, 1997), el dolor de miembro fantasma (Russell, 2007; Schneider et al., 2007, 2008; Wilensky, 2006), el síndrome de respuesta olfatoria (McGoldrick, Begum y Brown, 2008), y la activación sexual anormal (Ricci y Clayton, 2008) han sido resueltos a través del procesamiento de recuerdos centrales. Sin embargo, es necesaria la investigación controlada para tratar estos temas, comparando el tratamiento de los problemas presentados con el tratamiento de recuerdos centrales.

\section{Mecanismos sugeridos por los elementos del procedimiento EMDR durante la fase de evaluación}

Como indica Shapiro (2001), todas las formas complejas de psicoterapia poseen un rango de procedimientos y sus interacciones son responsables del resultado general del tratamiento. De ahí, como se ha señalado anteriormente, que sea demasiado simplista asumir que uno cualquiera de los mecanismos de acción fuese responsable de los efectos de EMDR. Por ejemplo, existen elementos del procedimiento, que concuerdan con el modelo PAI, que ocurren durante todas las fases de EMDR y que merecen la atención de la investigación en el análisis de los componentes para establecer su contribución relativa y medir el potencial mecanismo de acción contribuyente (ver Shapiro, 2001 para un debate más exhaustivo). 


\section{Selección de los blancos para el tratamiento}

La investigación de Mol y colegas (2005) comparó un rango de experiencias e informó que los eventos que no cumplen el criterio para ser designados como Criterio A del TEPT eran la causa de síntomas de trauma, similares a aquellos en el TEPT. Estos resultados ofrecen evidencia adicional de la predicción del PAI de que los eventos vitales perturbadores más ubicuos (traumas de "t pequeña") se encuentran almacenados de modo disfuncional y son la base de la patología. Por tanto, en un nivel de observación, se postula que el mecanismo de acción central inherente en EMDR es el procesamiento adaptativo del recuerdo. Funcionalmente, este procesamiento se logra accediendo al recuerdo almacenado, estimulando el sistema de procesamiento de la información de forma que permita que otras redes de memoria enlacen con la red disfuncional, transformando por tanto el recuerdo diana, y además haciendo blanco sobre los recuerdos en un orden que maximice el efecto terapéutico y la salud psicológica. En otras palabras: (a) acceso estructurado a los recuerdos con blancos sucesivos; (b) estimulación del sistema de procesamiento de la información a través de los elementos del procedimiento; y (c) fomentando la integración dinámica de otra información relevante.

Para maximizar el procesamiento adaptativo de la información, los recuerdos almacenados disfuncionalmente que parecen ser la base de los síntomas presentados han de ser identificados. Estos incluyen tanto traumas de $t$ pequeña como grande y disparadores del presente. Además, el clínico se asegura de que existan redes de memoria relacionadas y relevantes que contengan información positiva y/o adaptativa. Se postula que éstas son esenciales para que se puedan establecer enlaces apropiados durante el procesamiento. Se utiliza un protocolo estructurado que prepara al cliente, activa exhaustivamente el recuerdo perturbador y obtiene aspectos relevantes de la información almacenada disfuncionalmente.

\section{Mindfulness}

Las instrucciones que se dan a los clientes de "dejar que ocurra lo que ocurra" y de "simplemente notar" lo que aparece (Shapiro, 1989, 1995, 2001) concuerdan con los principios de lo que se conoce como "mindfulness" (atención consciente) (por ejemplo, Siegel, 2007). Dichas instrucciones no sólo reducen las características de demanda, sino que ayudan a los clientes a notar lo que están sintiendo y pensando, sin juzgarlo. La investigación ha mostrado la eficacia terapéutica de adaptar una tanda cognitiva en la que los pensamientos y emociones negativos sean vistos como eventos mentales pasajeros más que como aspectos del yo (por ejemplo, Teasdale, 1997; Teasdale et al., 2002). Teasdale (1997) señaló la importancia del proceso de "descentralización" o "desidentificación", durante el cual el cliente puede pasar de identificarse con la emoción a ver los pensamientos y emociones como pensamientos y emociones pasajeros que pueden o no ser ciertos. Esta separación cognitiva puede permitir a los clientes relacionarse con las experiencias negativas con un campo más amplio de conciencia, lo que puede aumentar su capacidad de afrontamiento (Beck, Rush, Shaw, \& Emery, 1979) y mejorar su confianza en su propia eficacia y dominio (Shapiro, 1995, 2001). Desde una perspectiva PAI, esta mayor capacidad de afrontamiento y autoconfianza es codificada en la red de memoria del cliente. Esto puede aumentar la habilidad del cliente de mantenerse presente con material dificil durante el procesamiento y ofrecer información positiva y adaptativa que está disponible para enlazarse con redes de memoria que contienen información almacenada disfuncionalmente. Además, los procedimientos de EMDR, incluyendo los concomitantes neurobiológicos de los movimientos oculares que dan como resultado una desactivación (Barrowcliff, Gray, Freeman, \& MacCulloch, 2004; Barrowcliff, Gray, MacCulloch, Freeman, \& Mac- Culloch, 2003; Elofsson, von Scheele, Theorell, \& Sondergaard, 2008) pueden producir el estado mental al que hace referencia Teasdale. Es necesario realizar investigación controlada para evaluar estas cuestiones.

\section{Alineación de los fragmentos de recuerdos}

Se postula que las experiencias que han sido insuficientemente procesadas se almacenan en fragmentos de recuerdo (van der Kolk \& Fisler, 1995). Por tanto, la alineación de los componentes del recuerdo parece ser un elemento del procedimiento que facilita el procesamiento. El protocolo EMDR implica la obtención de la imagen, la creencia negativa actual, la creencia positiva deseada, la emoción actual y la sensación física. Este procedimiento, que potencialmente alcanza diferentes partes del cerebro, permite la activación de diferentes aspectos de la información almacenada disfuncionalmente, los cuales se ha postulado que están codificados en diferentes redes de memoria, cada una de ellas con diferentes asociaciones y enlaces (Buckner \& Wheeler, 2001; Gottfried, Smith, Rugg, \& Dolan, 2004; Shapiro, 1995, 2001). La fase de evaluación alinea estos aspectos principales del recuerdo negativo, lo cual concuerda con el modelo BASK de disociación (en castellano CESC: comportamiento, 
emoción, sensación y conocimiento) (Braun, 1988). Este procedimiento de reconexión del material perturbador puede ayudar a los clientes a darle un sentido a la experiencia y facilitar el almacenamiento en la memoria narrativa.

\section{Conciencia somática}

Dirigir la atención del cliente a las sensaciones físicas, después de identificar la peor imagen o la más representativa, la cognición negativa y las emociones, también puede que sea un elemento del procedimiento relevante para obtener resultados positivos. Esto puede ayudar a los clientes a identificar y separar las sensaciones físicas de sus interpretaciones negativas, las cuales reflejan una sobre-identificación con sus emociones / sensaciones. Prestar atención a la sensación física y a la emoción separándolas de las interpretaciones negativas puede a ayudar al cliente a reconocer la mutabilidad de la sensación. Por ejemplo, el cliente puede pasar de identificarse con la emoción (Por ejemplo: "Tengo miedo") a reconocer que la experiencia de sensaciones en el estómago y en el pecho está asociada con el miedo. Esto puede aumentar la autoconfianza del cliente y su sentido de dominio (Shapiro, 1995, 2001), lo cual, desde una perspectiva PAI, aumenta la información positiva codificada en el cerebro disponible para enlazarse con redes de memoria que contienen información almacenada disfuncionalmente.

\section{Reestructuración cognitiva}

La reestructuración cognitiva es un elemento del procedimiento evidente en la fase de evaluación, que contribuye con otro posible mecanismo de acción, cuando el cliente identifica tanto cogniciones negativas como positivas. La identificación de la auto-creencia irracional y la reestructuración y reformulación de la creencia en una auto-creencia adaptativa puede facilitar el proceso terapéutico (Beck et al., 1979). Sin embargo, en la fase de evaluación de EMDR, no existen intentos específicos de cambiar o reformular la creencia que actualmente mantiene el cliente. Se asume que la creencia cambiará espontáneamente durante el curso del procesamiento posterior. No obstante, desde una perspectiva PAI, se cree que el forjar una asociación preliminar entre la cognición negativa y la información más adaptativa que contradiga la experiencia negativa facilita el procesamiento posterior por medio de la activación de redes adaptativas relevantes. El análisis de los procesos de los cambios espontáneos en el contenido cognitivo durante el tratamiento EMDR puede ayudar a evaluar la contribución del elemento cognitivo al resultado del tratamiento.

\section{Mecanismos sugeridos por los elementos del procedimiento EMDR durante las fases de desensibilización e instalación}

\section{Maestría percibida}

La maestría percibida puede ser otro importante elemento del procedimiento. Durante EMDR, los clientes pueden aumentar su sensación de dominio por su capacidad de limitar y manipular el material perturbador a través de las secuencias continuadas de acceso a la imaginería, la atención y la interrupción. Esto puede aumentar la eficacia del afrontamiento, lo cual puede mejorar la capacidad del cliente de manejar el estrés, la ansiedad y la depresión en situaciones amenazantes (Bandura, 2004). Desde una perspectiva PAI, la experiencia de dominio o maestría del cliente queda codificada en el cerebro como información adaptativa, disponible para enlazarse con redes de memoria que contienen información almacenada disfuncionalmente. Sería interesante que la investigación comparase la efectividad y eficacia de las condiciones que utilizan la estimulación bilateral, y a la vez comparase la atención continuada al material traumático con la atención interrumpida, como se hace en la práctica estándar de EMDR. Como es evidente en esta sección, queda como pregunta abierta el hecho de si el sentido de maestría o dominio aumenta debido a la secuencia, o si son simplemente la atención interrumpida junto a la estimulación bilateral, o una combinación de ambos, los principales elementos contribuyentes y mecanismos de cambio significativos.

\section{Efectos potenciales de los movimientos oculares}

Los procedimientos estructurados se utilizan para estimular las redes de memoria relevantes y establecer el procesamiento asociativo del cerebro durante las fases de desensibilización e instalación. De acuerdo con el modelo PAI, se accede a la información disfuncional tal y como está almacenada, y se aplica estimulación bilateral para ayudar en la estimulación del sistema de procesamiento intrínseco del cerebro, permitiendo que se enlace información de otras redes neurales. Se formula la hipótesis de que la creación de asociaciones es uno de los mecanismos que transmuta el recuerdo. Existe toda una serie de teorías acerca de cómo los movimientos oculares contribuyen a este proceso.

El modelo PAI considera los movimientos oculares y otras formas de estimulación bilateral como uno de los elementos que sirven para facilitar el procesamiento de la información. Desafortunadamente, 
los análisis de componentes aleatorios y controlados que se han realizado, utilizando poblaciones clínicas y medidas de resultados de tratamiento, eran defectuosos (ver Chemtob, Tolin, van der Kolk, \& Pitman, 2000; Shapiro, 2001) y necesitan ser realizados bajo condiciones más rigurosas. Sin embargo, hay un creciente número de investigaciones que han evaluado los movimientos oculares aislados con poblaciones no diagnosticadas (Gunter \& Bodner, 2008). Varios de estos estudios indican que los movimientos oculares producen un efecto de desensibilización durante el acceso a los recuerdos perturbadores. Por ejemplo, en un estudio de laboratorio, Barrowcliff et al. (2004) informaron que los movimientos oculares disminuyeron la activación fisiológica en las medidas electrodermales de conductancia de la piel.

Diversos estudios de tratamiento de TEPT (Elofsson et al., 2008; Sack, Hofmann, Wizelman, \& Lempa, este número; Sack, Lempa, \& Lemprecht, 2007; Sack, Lempa, Steinmetz, Lamprecht, \& Hofmann, 2008; Wilson et al, 1996), examinaron los efectos fisiológicos específicos de los movimientos oculares durante las sesiones de tratamiento EMDR. Los resultados sugirieron que los movimientos oculares dieron como resultado un aumento de la actividad parasimpática y un descenso en la activación psicofisiológica. Se encontraron resultados fisiológicos similares después de una sesión de EMDR, manifestados en un descenso del ritmo cardíaco y la conductancia de la piel (AubertKhalfa, Roques, \& Blin, 2008).

En otros estudios, se ha encontrado que los movimientos oculares reducen la intensidad y emocionalidad de los recuerdos negativos y positivos (Barrowcliff et al, 2004; Gunter \& Bodner, 2008; Kavanagh, Freese, Andrade, \& May, 2001; Maxfield, este número; Sharpley, Montgomery, \& Scalzo, 1996; van den Hout, Muris, Salemink, \& Kindt, 2001). En este momento, no sabemos si el cambio en intensidad precede o sigue a la desactivación fisiológica y si éstos ocurren conjuntamente o si son elementos separados. Sin embargo, se han adelantado toda una serie de hipótesis respecto al mecanismo de acción relacionado con la estimulación bilateral. Éstas incluyen la respuesta de orientación (MacCulloch \& Feldman, 1996), el sueño REM (movimientos oculares rápidos) (Stickgold, 2002, este número) y la memoria funcional (Andrad, Kavanagh, \& Baddeley, 1997). Todas estas hipótesis predicen los aparentes efectos de desensibilización presentes en diversos estudios. Es necesaria investigación adicional para identificar los mecanismos de acción reales y para determinar si existe una interacción entre los diversos mecanismos. Además, son necesarios estudios para evaluar la relación entre los cambios referidos y los resultados del tratamiento. En otras palabras, aún no sabemos el orden secuencial de estos efectos y no podemos asumir una causalidad. ¿Disminuye el ritmo cardíaco porque el recuerdo se hace menos perturbador debido al procesamiento, o el descenso de la activación facilita el procesamiento del recuerdo para que se vuelva menos perturbador? Tan solo la investigación aleatoria controlada, bajo condiciones apropiadas, puede zanjar estos interrogantes (ver Shapiro, 2001).

Con el descenso de la activación y la disminución de la intensidad y emocionalidad de los recuerdos negativos, la información de otras redes de memoria puede ser capaz de enlazarse con la red que contiene la información almacenada disfuncionalmente (ver Shapiro, 1995, 2001). Stickgold (2002) propone que los movimientos oculares utilizados en EMDR producen que se redirija la atención de manera repetitiva, lo cual induce un estado neurobiológico similar al sueño REM, que a su vez aumenta el acceso a asociaciones menos dominantes y podría resultar en una integración cortical de recuerdos perturbadores dentro de redes semánticas, reduciendo la fuerza de los recuerdos perturbadores. La transmutación del recuerdo parece incluir un cambio en la información sensorial que pasa de recuerdo implícito a episódico y después a semántico (Siegel, 2002; Stickgold, 2002).

El apoyo preliminar a los cambios en la recuperación de recuerdos proviene de Christman, Garvey, Propper y Phaneuf (2003) y Propper y Christman (este número), que muestran que los movimientos oculares mejoran la recuperación de recuerdos episódicos en estudios de laboratorio con participantes diestros no clínicos. Propper, Pierce, Geisler, Christman y Bellorado (2007) postulan que los movimientos oculares pueden cambiar la coherencia inter-hemisférica en las áreas frontales. Un estudio de Kuiken, Bears, Miall y Smith (2001-2002) encontró que los movimientos oculares estaban relacionados con un aumento de la flexibilidad de la atención. Es necesario investigar para replicar estos estudios en entornos clínicos con participantes diagnosticados zurdos y diestros. La investigación adicional debería evaluar la premisa de que la calidad del recuerdo diana se correlaciona con un aumento de los recuerdos no traumáticos asociativos. Esto ofrecería una oportunidad para comprobar la hipótesis generada por la investigación animal de Suzuki et al (2004). Ellos sugieren que, cuando se activa un recuerdo, parece volverse más voluble, con lo que la memoria puede reconsolidarlo de modo alterado. De ahí que sea posible que la reconsolidación ofrezca la habilidad, tal y como indican Przybyslawski, Roullet y Sara $(1999$, p. ), de permitir "la reorganización del 
recuerdo existente como una función de nueva información en el entorno de recuperación".

\section{Resumen y conclusión}

El modelo PAI (Shapiro, 1995, 2001) ha especificado que el recuerdo almacenado disfuncionalmente se ha transformado por medio del enlace con redes que contienen información adaptativa. Se ha postulado que este recuerdo se restaura en modo adaptativo. La implicación, desde una perspectiva PAI, es que las nuevas asociaciones enlazan con la red previamente aislada, causando una transmutación del propio recuerdo. Esto, a su vez, apoya el uso de procedimientos que fomentan un proceso asociativo interno. Aunque actualmente el campo de la neurobiología es incapaz de especificar el mecanismo por el que esto se consigue, la teoría de reconsolidación y la reciente investigación que la apoya parecen sugerir dicho mecanismo.

Cuando observamos los efectos de EMDR a través de las lentes de otros modelos dominantes del procesamiento de la información (por ejemplo, Foa \& McNally, 1996), la estimulación de asociaciones tal y como se lleva a cabo en EMDR sería considerado antitético a los efectos positivos del tratamiento porque fomentaría la evitación y simplemente reforzaría resultados conductuales y cognitivos negativos. Mientras que el modelo de procesamiento de información de Foa plantea específicamente la necesidad de acceder a la red del recuerdo disfuncional y la necesidad de incorporar información correctiva, el énfasis se sitúa en la alteración de la evaluación cognitiva de la experiencia a través de la exposición a la perturbación en un entorno terapéutico seguro. Como se menciona anteriormente, el PAI considera el cambio de las creencias no adaptativas como un subproducto del procesamiento, no el agente del cambio. Se considera que el mecanismo del cambio es la incorporación de información adaptativa a través de asociaciones internas con información que se encuentra ya almacenada en el cerebro. Sin embargo, como se mencionó anteriormente, muchos teóricos (por ejemplo Foa \& Kozak, 1986; Foa \& McNally, 1996; Marks, Lovell, Noshirvani, Livanou, \& Thrasher, 1998; Rothbaum \& Foa, 1996) suponen que la extinción es el mecanismo subyacente de la terapia de exposición prolongada. Y, como señalan Suzuki et al. (2004), se cree que el proceso de extinción produce otro recuerdo opuesto, no altera el original. De ahí, que los estudios que comparan el EMDR con las terapias de exposición basadas en la extinción puedan aclarar el proceso subyacente y determinar si de hecho el EMDR está basado en los efectos de reconsolidación de la memoria. Además de estudios que evaluaron los efectos en condiciones que se conjeturaría que causarían recaídas en los tratamientos basados en la extinción (ver Suzuki et al., 2004), sería útil observar si hay efectos diferenciales en los estudios de activación anormal y el dolor de un miembro fantasma, que han sido previamente considerados como condiciones intratables, pero sobre las que EMDR parece tener un impacto positivo (por ejemplo, Schneider et al., 2007, 2008).

El modelo PAI postula que el acceso simultáneo a la red del recuerdo traumático, combinado con la reducción de la angustia ocasionada por los movimientos de los ojos y los procedimientos usados para guiar la atención del cliente, nos lleva a un cambio integral del recuerdo, en el que las nuevas asociaciones son capaces de enlazarse con el recuerdo perturbador. Los elementos estructurados del procedimiento, así como la estimulación bilateral, son concebidos como efectos aditivos en el procesamiento adaptativo. Como señalaron Smyth, Rogers y Maxfield (2004), los resultados de estudios no publicados sugieren que los movimientos oculares parecen aumentar los efectos del tratamiento producidos únicamente por el resto de los procedimientos. Por ese motivo, son necesarias amplias muestras de poblaciones diagnosticadas apropiadas para determinar la contribución relativa de los diversos elementos (ver Shapiro, 2001, para un debate más exhaustivo). Los análisis de componentes previos que han utilizado resultados de tratamiento han demostrado ser defectuosos debido a la elección de poblaciones, la dosis de tratamiento y las mediciones de los resultados (Chemtob et al., 2000). Aunque los estudios de los movimientos oculares aislados han mostrado efectos pronunciados y significativos, dichos estudios necesitan ser replicados con participantes clínicos. El siguiente paso será determinar el valor y los concomitantes neurobiológicos de la estimulación bilateral en relación con el resultado del tratamiento. Es más, como se ha mencionado anteriormente, actualmente la investigación no ha determinado si la reducción en la intensidad de la imagen del recuerdo está relacionada con los cambios directos causados por el efecto de los movimientos oculares sobre la memoria funcional (Andrade et al., 1997) o si la imagen cambia por el efecto directo de los movimientos oculares sobre la activación fisiológica (Elofsson et al., 2008; Sack et al., 2007, 2008; Wilson et al., 1996).

Durante los últimos veinte años, EMDR ha evolucionado de una técnica de desensibilización hacia un enfoque psicoterapéutico integrador. El modelo PAI es la base teórica que integra los muchos elementos del procedimiento que contribuyen a los efectos del 
EMDR. Los problemas actuales, a no ser que tengan una base química o física, son debidos a experiencias pasadas que no han sido procesadas adecuadamente y están almacenadas de modo disfuncional. Aunque el modelo PAI no está ligado a un mecanismo neurobiológico específico, ofrece una explicación del cambio terapéutico como algo que se consigue a través del procesamiento de recuerdos disfuncionales y su integración dentro de redes adaptativas más amplias. Funcionalmente, esto se logra accediendo al recuerdo almacenado disfuncionalmente y estimulando el sistema de procesamiento de la información de modo que permita que otras redes de memoria se enlacen con la red disfuncional, lo cual transforma el recuerdo diana. Aunque los mecanismos precisos del cambio son desconocidos, los estudios muestran que los movimientos oculares utilizados en EMDR se correlacionan con un efecto de desensibilización, un aumento en la actividad parasimpática y un descenso de la activación psicofisiológica. De acuerdo con las investigaciones que muestran un aumento de la flexibilidad atencional y la recuperación de recuerdos, el descenso de la activación puede permitir que la información de otras redes de memoria enlace con la red que contienen la información almacenada disfuncionalmente. Sin embargo, es necesaria más investigación para determinar la causalidad de dichos efectos y los concomitantes biológicos de los movimientos oculares y otros tipos de estimulación utilizados en EMDR.

En última instancia, los mecanismos de acción son considerados como los facilitadores de la reorganización de las redes de memoria, con el modelo PAI guiando los procedimientos de EMDR necesarios para orquestar las actitudes clínicas, la conciencia del cliente y las conexiones neurobiológicas de los recuerdos codificados necesarios para conseguir estos objetivos. Por este motivo, se sugiere la realización de análisis de componentes con poblaciones diagnosticadas y condiciones de tratamiento y dosis que concuerden con el cuadro clínico (ver Shapiro, 2001, para un debate exhaustivo sobre parámetros de investigación). Comprobar las predicciones del PAI es un paso útil en la determinación de las condiciones clínicas apropiadas para comparar los mecanismos de cambio de los diversos enfoques psicoterapéuticos.

\section{References}

Andrade, J., Kavanagh, D., \& Baddeley, A. (1997). Eyemovement and visual imagery: A working memory approach to the treatment of post-traumatic stress dis- order. British Journal of Clinical Psychology, 36, 209-223.
Aubert-Khalfa, S., Roques, J., \& Blin, O. (2008). Evidence of a decrease in heart rate and skin conductance response in PTSD patients after a single EMDR session. Journal of EMDR Practice and Research, 2, 51-56.

Bandura, A. (2004). Swimming against the mainstream: The early years from chilly tributary to transformative mainstream. Behaviour Research and Therapy, 42, 613-630.

Barrowcliff, A. L., Gray, N. S., Freeman, T. C. A., \& MacCulloch, M. J. (2004). Eye-movements reduce the vividness, emotional valence and electrodermal arousal associated with negative autobiographical memories. Journal of Forensic Psychiatry and Psychology, 15, 323-345.

Barrowcliff, A. L., Gray, N. S., MacCulloch, S., Freeman, T.C.A., \& MacCulloch, M. J. (2003). Horizontal rhythmical eye movements consistently diminish the arousal provoked by auditory stimuli. British Journal of Clinical Psychology, 42, 289-302.

Beck, A. T., Rush, A. J., Shaw, B. F., \& Emery, G. (1979). Cognitive therapy of depression. New York: Guilford Press.

Braun, B. G., (1988). The BASK model of dissociation. Dissociation, 1, 4-23.

Brown, K. W., McGoldrick, T., \& Buchanan, R. (1997). Body dysmorphic disorder: Seven cases treated with eye movement desensitization and reprocessing. Behavioural \& Cognitive Psychotherapy, 25, 203-207.

Buchanon, T. W. (2007). Retrieval of emotional memories. Psychological Bulletin, 5, 761-779.

Buckner, R. L., \& Wheeler, M. E. (2001). The cognitive neuroscience of remembering. Nature Reviews Neuroscience, 2, 624-634.

Brewin, C. R. (2006). Understanding cognitive-behaviour therapy: A retrieval competition account. Behaviour Research and Therapy, 44, 765-784.

Cahill, L., \& McGaugh, J. L. (1998). Mechanisms of emotional arousal and lasting declarative memory. Trends in Neuroscience, 21, 294-299.

Chemtob, C. M., Tolin, D. F., van der Kolk, B. A., \& Pitman, R. K. (2000). Eye movement desensitization and reprocessing. In E. A. Foa, T. M. Keane, \& M. J. Friedman (Eds.), Effective treatments for PTSD: Practice guidelines from the International Society for Traumatic Stress Studies (pp. 139-155, 333-335). New York: Guilford Press.

Christman, S. D., Garvey, K. J., Propper, R. E., \& Phaneuf, K. A. (2003). Bilateral eye movements enhance the retrieval of episodic memories. Neuropsychology, 17, 221-229.

Elofsson, U.O.E., von Scheele, B., Theorell, T., \& Sondergaard, H. P. (2008). Physiological correlates of eye movement desensitization and reprocessing. Journal of Anxiety Disorders, 622-624.

Foa, E. B., \& Kozak, M. J. (1986). Emotional processing of fear: Exposure to corrective information. Psychological Bulletin, 99(1), 20-35.

Foa, E. B., \& McNally, R. J. (1996). Mechanisms of change in exposure therapy. In R. M. Rapee (Ed.), Current controversies in the anxiety disorders (pp. 329-343). New York: Guilford Press. 
Foa, E. B., Rothbaum, B. O., Riggs, D. S., \& Murdock, T. B. (1991). Treatment of posttraumatic stress disorder in rape victims: A comparison between cognitivebehavioral procedures and counseling. Journal of Consulting and Clinical Psychology, 59, 715-723.

Gottfried, J. A., Smith, A. P., Rugg, M. D., \& Dolan, R. J. (2004). Remembrance of odors past: Human olfactory cortex in cross-modal recognition memory. Neuron, 42, 687-695.

Gunter, R. W., \& Bodner, G. E. (2008). How eye movements affect unpleasant memories: Support for a working memory account. Behaviour Research and Therapy, 46, 913-931.

Hofmann, S. G., Meuret, A. E., Smits, J. A., Simon, N. M., Pollack, M. H., et al. (2006). Augmentation of exposure therapy with D-cycloserine for social anxiety disorder. Archives of General Psychiatry, 63, 298-304.

Kavanagh, D. J., Freese, S., Andrade, J., \& May, J. (2001). Effects of visuospatial tasks on desensitization to emotive memories. British Journal of Clinical Psychology, 40, 267-280.

Kuiken, D., Bears, M., Miall, D., \& Smith, L. (2001-2002). Eye movement desensitization reprocessing facilitates attentional orienting. Imagination, Cognition and Personality, 21, 3-20.

Lee, C., Taylor, G., \& Drummond, P. D. (2006). The active ingredient in EMDR: Is it traditional exposure or dual focus of attention? Clinical Psychology and Psychotherapy, 13, 97-107.

Lee, J. L., Milton, A. L., \& Everitt, B. J. (2006). Reconsolidation and extinction of conditioned fear: Inhibition and potentiation. Journal of Neuroscience, 26(39), 10051-10056.

Marks, I., Lovell, K., Noshirvani, H., Livanou, M., \& Thrasher, S. (1998). Treatment of posttraumatic stress disorder by exposure and/or cognitive restructuring. Archives of General Psychiatry, 55, 317-325.

MacCulloch, M. J., \& Feldman, P. (1996). Eye movement desensitization treatment utilizes the positive visceral element of the investigatory reflex to inhibit the memories of post-traumatic stress disorder: A theoretical analysis. British Journal of Psychiatry, 169, 571-579.

McCleery, J. M., \& Harvey, A. G. (2004). Integration of psychological and biological approaches to trauma memory: Implications for pharmacological prevention of PTSD. Journal of Traumatic Stress, 17, 485-496.

McCullough, L. (2002). Exploring change mechanisms in EMDR applied to "small t trauma" in short term dynamic psychotherapy: Research questions and speculations. Journal of Clinical Psychology, 58, 1465-1487.

McGoldrick, T., Begum, M., \& Brown, K. W. (2008). EMDR and olfactory reference syndrome: A case series. Journal of EMDR Practice and Research, 2, 63-68.

Mol, S.S.L., Arntz, A., Metsemakers, J. F. M., Dinant, G., Vilters-Van Montfort, P. A. P., \& Knottnerus, A. (2005). Symptoms of post-traumatic stress disorder after nontraumatic events: Evidence from an open population study. British Journal of Psychiatry, 186, 494-499.
Propper, R., Pierce, J. P., Geisler, M. W., Christman, S. D., \& Bellorado, N. (2007). Effect of bilateral eye movements on frontal interhemispheric gamma EEG coherence: Implications for EMDR therapy. Journal of Nervous and Mental Disease, 195, 785-788.

Przybyslawski, J., Roullet, P., \& Sara, S. J. (1999). Attenuation of emotional and non-emotional memories after their reactivation: Role of â adrenergic receptors. Journal of Neuroscience, 19, 6623-6628.

Ressler, K. J., Rothbaum, B. O., Tannenbaum, L., Anderson, P., Graap, K., et al. (2004). Cognitive enhancers as adjuncts to psychotherapy: Use of D-cycloserine in phobic individuals to facilitate extinction of fear. Archives of General Psychiatry, 61, 1136-1144.

Ricci, R. J., \& Clayton, C. A. (2008). Trauma resolution treatment as an adjunct to standard treatment for child molesters: A qualitative study. Journal of EMDR Practice and Research, 1, 41-50.

Rogers, S., \& Silver, S. M. (2002). Is EMDR an exposure therapy? A review of trauma protocols. Journal of Clinical Psychology, 58, 43-59.

Rogers, S., Silver, S., Goss, J., Obenchain, J., Willis, A., \& Whitney, R. (1999). A single session, controlled group study of flooding and eye movement desensitization and reprocessing in treating posttraumatic stress disorder among Vietnam war veterans: Preliminary data. Journal of Anxiety Disorders, 13, 119-130.

Rost, C., Hofmann, A., \& Wheeler, K. (in press). EMDR treatment of workplace trauma: A case series. Journal of EMDR Practice and Research.

Rothbaum, B. O., Astin, M. C., \& Marsteller, F. (2005). Prolonged exposure versus eye movement desensitization (EMDR) for PTSD rape victims. Journal of Traumatic Stress, 18, 607-616.

Rothbaum, B. O., \& Foa, E. B. (1996). Cognitive-behavioral therapy for posttraumatic stress disorder. In B. A. van der Kolk, A. C. McFarlane, \& L. Weisaeth, (Eds.), Traumatic stress: The effects of overwhelming experience on mind, body and society (pp. 491-509). New York: Guilford Press.

Russell, M. (2008). Treating traumatic amputation-related phantom limb pain: A case study utilizing eye movement desensitization and reprocessing (EMDR) within the armed services. Clinical Case Studies, 7, 136-153.

Russell, M. C., Silver, S. M., Rogers, S., \& Darnell, J. N. (2007). Responding to an identified need: A joint Department of Defense/Department of Veterans Affairs training pro- gram in eye movement desensitization and reprocessing (EMDR) for clinicians providing trauma services. International Journal of Stress Management, 14(1), 61.

Sack, M., Lempa, W., \& Lemprecht, W. (2007). Assessment of psychophysiological stress reactions during a traumatic reminder in patients treated with EMDR. Journal of EMDR Practice and Research, 1, 15-23.

Sack, M., Lempa, W., Steinmetz, A., Lamprecht, F., \& Hofmann, A. (2008). Alterations in autonomic tone during trauma exposure using eye movement desensitization 
and reprocessing (EMDR): Results of a preliminary investigation. Journal of Anxiety Disorders, 22(7), 1264-1271.

Schneider, J., Hofmann, A., Rost, C., \& Shapiro, F. (2007). EMDR and phantom limb pain: Case study, theoretical implications, and treatment guidelines. Journal of EMDR Practice and Research, 1, 31-45.

Schneider, J., Hofmann, A., Rost, C., \& Shapiro, F. (2008). EMDR in the treatment of chronic phantom limb pain. Pain Medicine, 9, 76-82.

Shapiro, F. (1989). Efficacy of the eye movement desensitization procedure in the treatment of traumatic memories. Journal of Traumatic Stress Studies, 2, 199-223.

Shapiro, F. (1995). Eye movement desensitization and reprocessing: Basic principles, protocols, and procedures. New York: Guilford Press.

Shapiro, F. (2001). Eye movement desensitization and reprocessing: Basic principles, protocols, and procedures (2nd ed.). New York: Guilford Press.

Shapiro, F. (2002) Paradigms, processing, and personality development. In F. Shapiro (Ed.), EMDR as an integrative psychotherapy approach: Experts of diverse orientations explore the paradigm prism (pp. 3-26). Washington, DC: American Psychological Association Press.

Shapiro, F. (2006). New notes on adaptive information processing. Hamden, CT: EMDR Humanitarian Assistance Programs.

Shapiro, F. (2007a). EMDR and case conceptualization from an adaptive information processing perspective. In F. Shapiro, F. Kaslow, \& L. Maxfield (Eds.), Handbook of EMDR and family therapy processes (pp. 3-36). New York: Wiley.

Shapiro, F. (2007b, September). EMDR update: Theory, research, and practice. Keynote presentation at the EMDR International Association annual conference, Dallas, TX.

Shapiro, F. \& Forrest, M. S. (1997). EMDR: The breakthrough therapy for overcoming anxiety, stress, and trauma. New York: Basic Books.

Shapiro, F., Kaslow, F., \& Maxfield, L. (Eds.). (2007). Handbook of EMDR and family therapy processes. New York: Wiley.

Sharpley, C. F., Montgomery, I. M., \& Scalzo, L. A. (1996). Comparative efficacy of EMDR and alternative procedures in reducing the vividness of mental images. Scandinavian Journal of Behaviour Therapy, 25, 37-42.

Siegel, D. J. (2002). The developing mind and the resolution of trauma: Some ideas about information processing and an interpersonal neurobiology of psychotherapy.
In F. Shapiro (Ed.), EMDR as an integrative psychotherapy approach: Experts of diverse orientations explore the paradigm prism (pp. 85-122). Washington, DC: American Psychological Association Press.

Siegel, D. J. (2007). The mindful brain. New York: Norton.

Smyth, N. J., Rogers, S., \& Maxfield, L. (2004, September). What about eye movements? Plenary at the annual conference of the EMDR International Association, Montreal,

Canada. Stickgold, R. (2002). EMDR: A putative neurobiological mechanism of action. Journal of Clinical Psychology, 58, 61-75.

Suzuki, A., Josselyn, S. A., Frankland, P. W., Masushige, S., Silva, A. J., \& Kida, S. (2004). Memory reconsolidation and extinction have distinct temporal and biochemical signatures. Journal of Neuroscience, 24, 4787- 4795.

Teasdale, J. D. (1997). The relationship between cognition and emotion: The mind-in-place in mood disorders. En D.M. Clark \& C. G. Fairburn (Eds.), Science and practice of cognitive behaviour therapy (pp. 67-93). Oxford: Oxford University Press.

Teasdale, J. D., Moore, R. G., Hayhurst, H., Pope, M., Williams, S., \& Segal, Z. (2002). Metacognitive awareness and prevention of relapse in depression: Empirical evidence. Journal of Consulting and Clinical Psychology, 70, 275-287.

van den Hout, M., Muris, P., Salemink, E., \& Kindt, M. (2001). Autobiographical memories become less vivid and emotional after eye movements. British Journal of Clinical Psychology, 40, 121-130.

van der Kolk, B. A. \& Fisler, R. (1995). Dissociation and the fragmentary nature of traumatic memories: Overview and exploratory study. Journal of Traumatic Stress, $8,505-525$.

Wilensky, M. (2006). Eye movement desensitization and reprocessing (EMDR) as a treatment for phantom limb pain. Journal of Brief Therapy, 5, 31-44.

Wilson, D., Silver, S. M., Covi, W., \& Foster, S. (1996). Eye movement desensitization and reprocessing: Effectiveness and autonomic correlates. Journal of Behaviour Therapy and Experimental Psychiatry, 27, 219-229.

Zaghout-Hodali, M., Alissa, F., \& Dodgson, P. (2008). EMDR and resilience in children in ongoing trauma. Journal of EMDR Practice and Research, 1, 106-113.

Correspondencia respecto a este artículo deberá dirigirse a Dr. Roger M. Solomon, 1813 Northwood Drive, Williamsville, NY 14221. E-mail: Rogermsolomon@aol.com 